1 Universidade Federal de Santa Catarina (UFSC), Centro de Ciências da Saúde, Programa de Pós-Graduação em Saúde Coletiva - Florianópolis (SC), Brasil.

karinenfe@hotmail.com

2 Universidade Federal de Santa Catarina (UFSC),

Centro de Ciências da

Saúde, Programa de

Pós-Graduação em Saúde Coletiva - Florianópolis

(SC), Brasil.

jtelino@gmail.com

3 Universidade Federal de Santa Catarina (UFSC)

Centro de Ciências da

Saúde, Programa de

Pós-Graduação em Saúde

Coletiva - Florianópolis

(SC), Brasil.

patriciamomachado@gmail. com

\section{O processo de trabalho na Atenção Básica à saúde: avaliação da gestão}

Work Process in Primary Health Care: evaluation of management

Karine Cardoso Fontana', Josimari Telino de Lacerda² ${ }^{2}$ Patrícia Maria de Oliveira Machado $\mathbf{3}$

RESUMO Esta pesquisa desenvolveu e aplicou um modelo de avaliação da gestão do processo de trabalho na Atenção Básica à saúde em municípios catarinenses, utilizando os dados do segundo ciclo da avaliação externa do Programa Nacional de Melhoria do Acesso e da Qualidade da Atenção Básica. O universo avaliado correspondeu a 91,9\% dos municípios do estado de Santa Catarina que, em sua maioria, apresentaram resultados satisfatórios nas condições estruturais para o processo de trabalho. Porém, existem grandes desafios para assegurar as condições necessárias à realização dos aspectos processuais, em especial, na continuidade do cuidado e na organização do trabalho.

PALAVRAS-CHAVE Atenção Primária à Saúde. Avaliação em saúde. Avaliação de processos (cuidados de saúde).

ABSTRACT This research has developed and implemented an evaluation model of the management of the work process in Primary Health Care in municipalities of Santa Catarina, using the data of the second cycle of the external evaluation of the National Program for Access and Quality Improvement in Primary Care. The rated universe corresponded to $91.9 \%$ of the municipalities of Santa Catarina that, mostly, achieved satisfactory outcomes on structural conditions for the working process. However, there are great challenges to ensure the necessary conditions for the realization of procedural aspects, in particular, on the continuity of care and on the work organization.

KEYWORDS Primary Health Care. Health evaluation. Process assessment (health care). 


\section{Introdução}

O modelo de Atenção Primária em Saúde (APS) é desenvolvido, no Brasil, por meio da Estratégia Saúde da Família (ESF), sendo baseado na atenção integral à saúde, com vínculo das equipes multiprofissionais à população de um determinado território; possui ampla capilaridade; é considerada a porta de entrada preferencial para o sistema e responsável pela coordenação das redes de atenção à saúde (SUMAR; FAUSTO, 2014).

Para efetivar todos os pressupostos a que se propõe, o processo de trabalho em saúde das Equipes de Atenção Básica (EqAB) deve contemplar um conjunto de ações coordenadas e orientadas, de caráter individual e coletivo, desde a promoção à reabilitação da doença, a fim de modificar a situação de saúde da população, seus determinantes e condicionantes (BRASIL, 2011B). Mesmo com a ampliação do acesso e a estruturação dos serviços de Atenção Básica (AB), existem muitos desafios enfrentados pela gestão para efetivar a mudança do modelo de atenção à saúde no País (PAIM ET AL., 2011).

O processo de trabalho em saúde é entendido como um conjunto de ações coordenadas, desenvolvidas pelos trabalhadores, onde indivíduos, famílias e grupos sociais compõem o objeto de trabalho, e os saberes e métodos representam os instrumentos que originam a atenção em saúde. Os modelos de atenção que orientam o trabalho em saúde refletem as combinações tecnológicas para o alcance dos objetivos, pois são "[...] uma espécie de lógica que orienta a ação e organiza os meios de trabalho (saberes e instrumentos) utilizados nas práticas de saúde" (PAIM, 2008, P. 554).

No âmbito municipal, a gestão dos serviços de saúde assume significado estratégico para a consolidação do processo de trabalho das equipes com a reorientação de um modelo de saúde baseado nas necessidades da população (FERREIRA, 2012). A implantação de mecanismos que monitorem as condições necessárias para execução do processo de trabalho torna-se fundamental, uma vez que o serviço prestado pelas equipes depende diretamente de suporte administrativo, da adequação de estrutura e organização das ações consoantes com os princípios do Sistema Único de Saúde (SUS).

Desde 2003, o Ministério da Saúde tem desenvolvido ações com vistas à institucionalização da avaliação no âmbito da $\mathrm{AB}$. A iniciativa mais recente para a implantação de processos avaliativos sistemáticos sobre a atuação das equipes no âmbito da $\mathrm{AB}$ foi o Programa Nacional de Melhoria do Acesso e da Qualidade da Atenção Básica (PMAQ-AB), cujo objetivo é avaliar aspectos estruturais oferecidos pela gestão municipal, aspectos do processo de trabalho desenvolvidos pelas equipes e de satisfação do usuário. Ao emitir o julgamento, o faz de maneira global, com foco na qualidade da atenção e no acesso aos serviços (BRASIL, 2011A).

O presente estudo desenvolveu e aplicou um modelo de avaliação do processo de trabalho na $A B$ em saúde com foco na gestão municipal.

\section{Métodos}

Trata-se de uma pesquisa avaliativa das ações da gestão municipal do processo de trabalho na AB. O estudo adotou como elemento avaliativo os municípios catarinenses que participaram da avaliação externa do PMAQ, em 2013, e como unidade de análise, as EqAB. Foram incluídos os municípios que aderiram ao PMAQ com, pelo menos, $80 \%$ das suas equipes, totalizando 271 municípios e 1.399 equipes da $\mathrm{AB}$.

A pesquisa avaliativa foi realizada em duas etapas: construção do modelo de avaliação e classificação dos municípios quanto à gestão municipal do processo de trabalho na $\mathrm{AB}$.

$\mathrm{Na}$ construção do modelo de avaliação foram elaborados e definidos o modelo teórico-lógico e a Matriz Avaliativa e de Julgamento (MAJ). O modelo teórico-lógico baseou-se em pesquisa documental e bibliográfica. A pesquisa documental foi 
composta por portarias e legislação pertinentes ao tema. A revisão bibliográfica adotou as bases de dados do Centro Latino-Americano e do Caribe de Informação em Ciências da Saúde (Bireme), da Literatura Latino-Americana e do Caribe em Ciências da Saúde (Lilacs) e da Scientific Electronic Library Online (SciELO), com a palavra-chave 'Processo de Trabalho' associado aos descritores 'Atenção Primária à Saúde', 'Avaliação em Saúde' e 'Sistema Único de Saúde'. Como critério de inclusão, foram utilizados artigos de abordagem total ou parcial da gestão municipal do processo de trabalho na $\mathrm{AB}$, sendo encontrados 47 artigos e selecionados 26 que apresentavam reflexões teóricas e experiências da gestão municipal do processo de trabalho na $\mathrm{AB}$, de forma descritiva ou avaliativa, sem limitação cronológica, em português, espanhol e inglês.

A revisão bibliográfica buscou identificar os indicadores, medidas e parâmetros que conformaram uma proposta inicial da MAJ para análise dos especialistas. Foram realizadas oficinas de consenso presenciais, que possibilitaram a discussão acerca das dimensões, dos critérios e indicadores propostos previamente, com o objetivo de promover a troca de experiências e o confronto de opiniões, principalmente, em torno dos pontos de maior divergência. Para as oficinas de consenso, foram convidados especialistas na área de avaliação em saúde e com familiaridade teórica e prática em relação ao processo de trabalho na AB. Como estratégia, enviou-se, previamente, aos especialistas uma proposta de matriz para análise e emissão de opinião acerca de sua concordância plena, parcial ou não concordância com cada um dos elementos propostos. Foram consolidadas as análises, acatando as concordâncias plenas e apresentando os dissensos em outros dois encontros presenciais, nos quais as decisões foram tomadas por consenso.

O modelo de avaliação final conta com 22 indicadores, 2 dimensões e 6 subdimensões. A dimensão 'Condições Estruturais' aborda aspectos de 'Infraestrutura', 'Pessoal' e 'Apoio Tecnológico e Logístico' para que as equipes realizem suas atividades. A dimensão 'Condições Processuais' analisa aspectos do processo de trabalho das equipes da $\mathrm{AB}$, agrupados nas subdimensões: 'Vínculo e Territorialização', 'Organização do Trabalho' e 'Coordenação do Cuidado'. O detalhamento dos componentes da matriz de análise está apresentado no quadro 1.

\begin{tabular}{|c|c|c|c|c|}
\hline \multicolumn{2}{|c|}{ Matriz avaliativa } & \multirow{2}{*}{$\begin{array}{l}\text { Medidas } \\
\text { \% UBSs com sala de observação }\end{array}$} & \multicolumn{2}{|l|}{ Parâmetros } \\
\hline \multirow{7}{*}{ 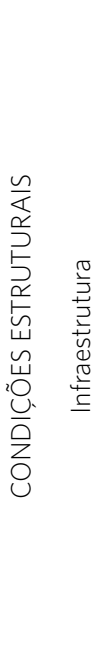 } & \multirow{3}{*}{$\begin{array}{l}\text { Espaço físico } \\
\text { adequado para o } \\
\text { desenvolvimento } \\
\text { das ações }\end{array}$} & & $\begin{array}{l}\text { 100\%: BOM } \\
<100 \%: \text { RUIM }\end{array}$ & \multirow{5}{*}{$\begin{array}{l}\text { Ambas bom: } B O M \\
\text { Demais: REG } \\
\text { Ambas ruim: RUIM }\end{array}$} \\
\hline & & \multirow[t]{2}{*}{$\%$ UBSs com sala de atividades coletivas } & $\begin{array}{l}\text { 3quartil: BOM } \\
\text { INTERV: REG }\end{array}$ & \\
\hline & & & $1^{\circ}$ quartil: RUIM & \\
\hline & $\begin{array}{l}\text { Existência } \\
\text { de suporte } \\
\text { tecnológico }\end{array}$ & $\begin{array}{l}\text { \% UBSs com, no mínimo, um consultório } \\
\text { com computador que acesse à Internet }\end{array}$ & $\begin{array}{l}\text { 290\%: BOM } \\
\text { 89\% a } 75 \%: \text { REG } \\
<75 \%: \text { RUIM }\end{array}$ & \\
\hline & $\begin{array}{l}\text { Recursos de } \\
\text { mobilidade da } \\
\text { equipe }\end{array}$ & $\begin{array}{l}\% \text { UBSs com disponibilidade de veículo que } \\
\text { responda às necessidades da equipe }\end{array}$ & $\begin{array}{l}\text { 3q quartil: BOM } \\
\text { INTERV: REG } \\
\text { 1ㅇ quartil: RUIM }\end{array}$ & \\
\hline & \multirow[t]{2}{*}{$\begin{array}{l}\text { Disponibilidade } \\
\text { para o acesso }\end{array}$} & $\begin{array}{l}\text { Existência de UBSs funcionando em horário } \\
\text { noturno }\end{array}$ & $\begin{array}{l}\text { Sim: BOM } \\
\text { Não: RUIM }\end{array}$ & \multirow[t]{2}{*}{$\begin{array}{l}\text { Ambas bom: } B O M \\
\text { Demais: REG } \\
\text { Ambas ruim: RUIM }\end{array}$} \\
\hline & & $\%$ UBSs que funcionam $40 \mathrm{~h} / \mathrm{semana}$ & $\begin{array}{l}\geq 90 \%: \text { BOM } \\
<90 \%: \text { RUIM }\end{array}$ & \\
\hline
\end{tabular}




\section{Quadro 1. (cont.)}

\begin{tabular}{|c|c|c|c|c|}
\hline \multirow{3}{*}{ 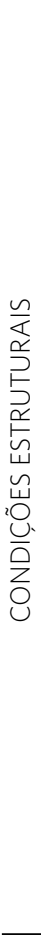 } & \multirow[b]{2}{*}{$\begin{array}{l}\bar{N} \\
0 \\
\sim \\
\sim \\
0 \\
0\end{array}$} & $\begin{array}{l}\text { Cobertura } \\
\text { populacional** }^{\star *}\end{array}$ & $\begin{array}{l}\text { \% população com cobertura da equipe de } \\
A B\end{array}$ & $\begin{array}{l}\text { Até } 10 \text { mil habitantes }=100 \%: \text { BOM } \\
\text { Entre } 10 \text { e } 50 \text { mil habitantes }=80 \% \text { : } \\
\text { BOM } \\
\text { Acima de } 50 \text { mil habitantes }=60 \% \text { : } \\
\text { BOM }\end{array}$ \\
\hline & & $\begin{array}{l}\text { Adequação de } \\
\text { pessoal } \\
\text { Suporte } \\
\text { especializado* }\end{array}$ & $\begin{array}{l}\text { \% equipes da } A B \text { que recebe apoio de ou- } \\
\text { tros profissionais para auxiliar na resolução } \\
\text { de casos considerados complexos } \\
\text { \% equipes da AB que participam do Teles- } \\
\text { saúde e/ou da Universidade Aberta do SUS } \\
\text { (UNASUS) e/ou de troca de experiências }\end{array}$ & $\begin{array}{l}\text { 100\%: BOM } \\
<100 \%: \text { RUIM } \\
\text { 3 quartil: BOM } \\
\text { INTERV: REG } \\
\text { 1quartil: RUIM } \\
\text { 3 quartil: BOM } \\
\text { INTERV: REG } \\
\text { 1 quartil: RUIM }\end{array}$ \\
\hline & 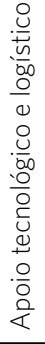 & $\begin{array}{l}\text { Orientação } \\
\text { de fluxo de } \\
\text { encaminhamento } \\
\text { Normatização do } \\
\text { cuidado }\end{array}$ & $\begin{array}{l}\text { \% equipes em que, na maioria das vezes, } \\
\text { o encaminhamento é realizado através de } \\
\text { central de regulação } \\
\text { \% equipes que utilizam protocolos para } \\
\text { estratificação de risco: câncer do colo do } \\
\text { útero, câncer da mama, hipertensão arterial } \\
\text { sistêmica, diabetes mellitus, tuberculose, } \\
\text { saúde mental }\end{array}$ & 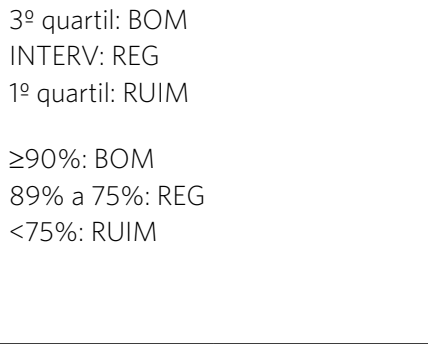 \\
\hline \multirow{3}{*}{ 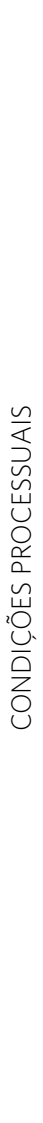 } & 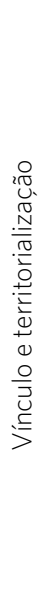 & $\begin{array}{l}\text { Participação } \\
\text { popular nas } \\
\text { decisões }\end{array}$ & $\begin{array}{l}\text { \% equipes da } A B \text { que dispõem de canais } \\
\text { de comunicação para que os usuários } \\
\text { expressem suas demandas, reclamações e/ } \\
\text { ou sugestões } \\
\text { \% equipes da AB que possuem Conselho } \\
\text { Local de Saúde ou outros espaços de parti- } \\
\text { cipação popular }\end{array}$ & $\begin{array}{l}\geq 90 \%: \text { BOM } \\
\text { 89\% a } 75 \%: \text { REG } \\
<75 \%: \text { RUIM } \\
\text { 3 quartil: BOM } \\
\text { INTERV: REG } \\
\text { 1q quartil: RUIM }\end{array}$ \\
\hline & \multirow[b]{2}{*}{ 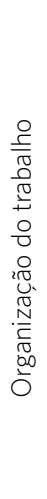 } & Foco na família & $\begin{array}{l}\text { \% equipes da } \mathrm{AB} \text { com prontuário organiza- } \\
\text { do pelo núcleo familiar }\end{array}$ & $\begin{array}{l}\geq 90 \%: \text { BOM } \\
89 \% \text { a } 75 \%: \text { REG } \\
<75 \%: \text { RUIM }\end{array}$ \\
\hline & & $\begin{array}{l}\text { Planejamento } \\
\text { conjunto } \\
\text { Ações } \\
\text { compartilhadas }\end{array}$ & $\begin{array}{l}\text { \% equipes da } A B \text { que atuam junto aos } \\
\text { profissionais de educação para atividades } \\
\text { de prevenção e promoção em saúde } \\
\text { \% equipes da } A B \text { que se reúnem mensal- } \\
\text { mente para planejar suas ações } \\
\text { \% equipes da AB que possuem uma agen- } \\
\text { da de atividades organizada pelo conjunto } \\
\text { de seus profissionais }\end{array}$ & $\begin{array}{l}\geq 90 \%: \text { BOM } \\
89 \% \text { a } 75 \%: \text { REG } \\
<75 \%: \text { RUIM } \\
\text { 100\%: BOM } \\
<100 \%: \text { RUIM } \\
\geq 90 \%: \text { BOM } \\
\text { 89\% a } 75 \%: \text { REG } \\
<75 \%: \text { RUIM }\end{array}$ \\
\hline
\end{tabular}




\section{Quadro 1. (cont.)}

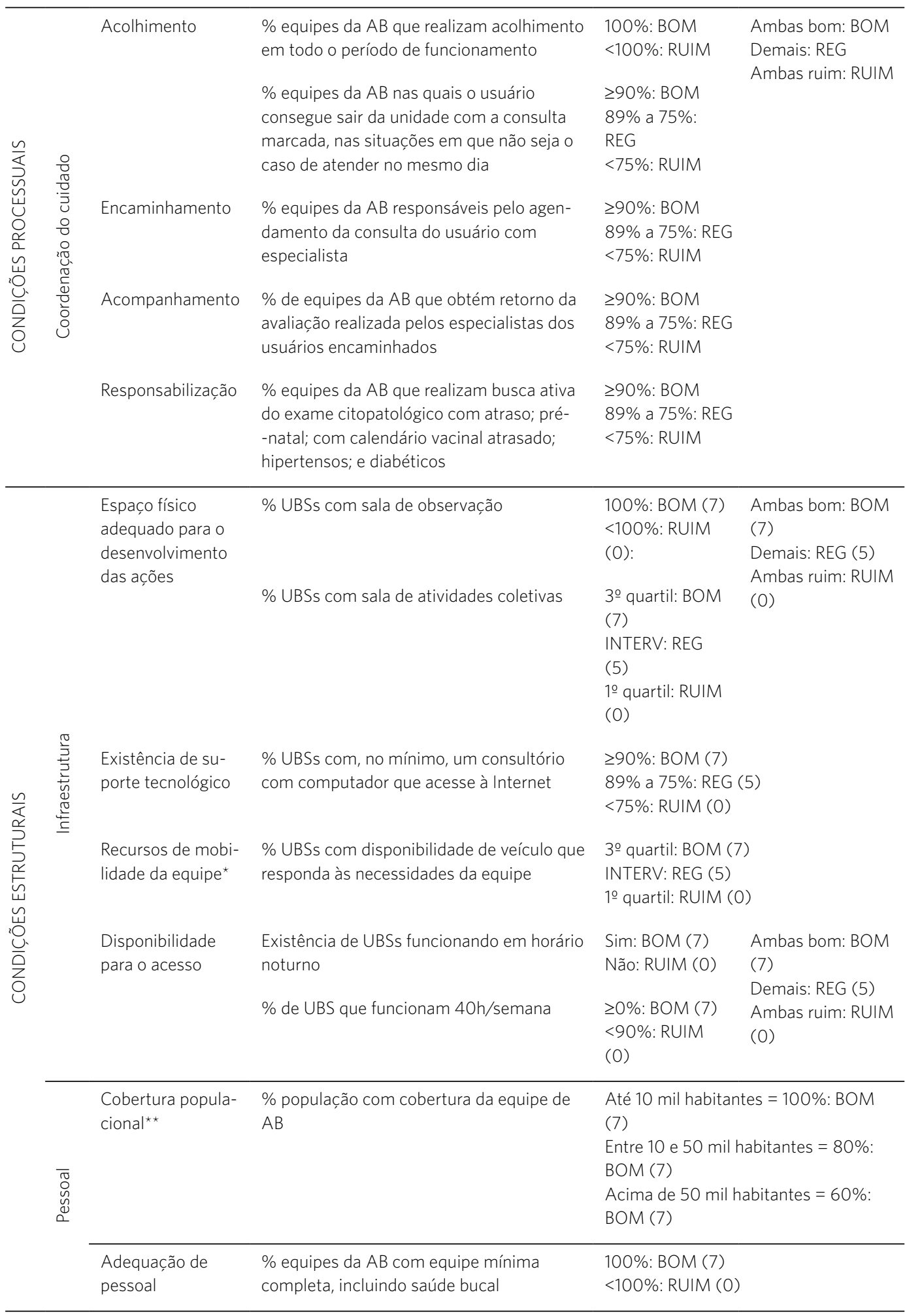


Quadro 1. (cont.)

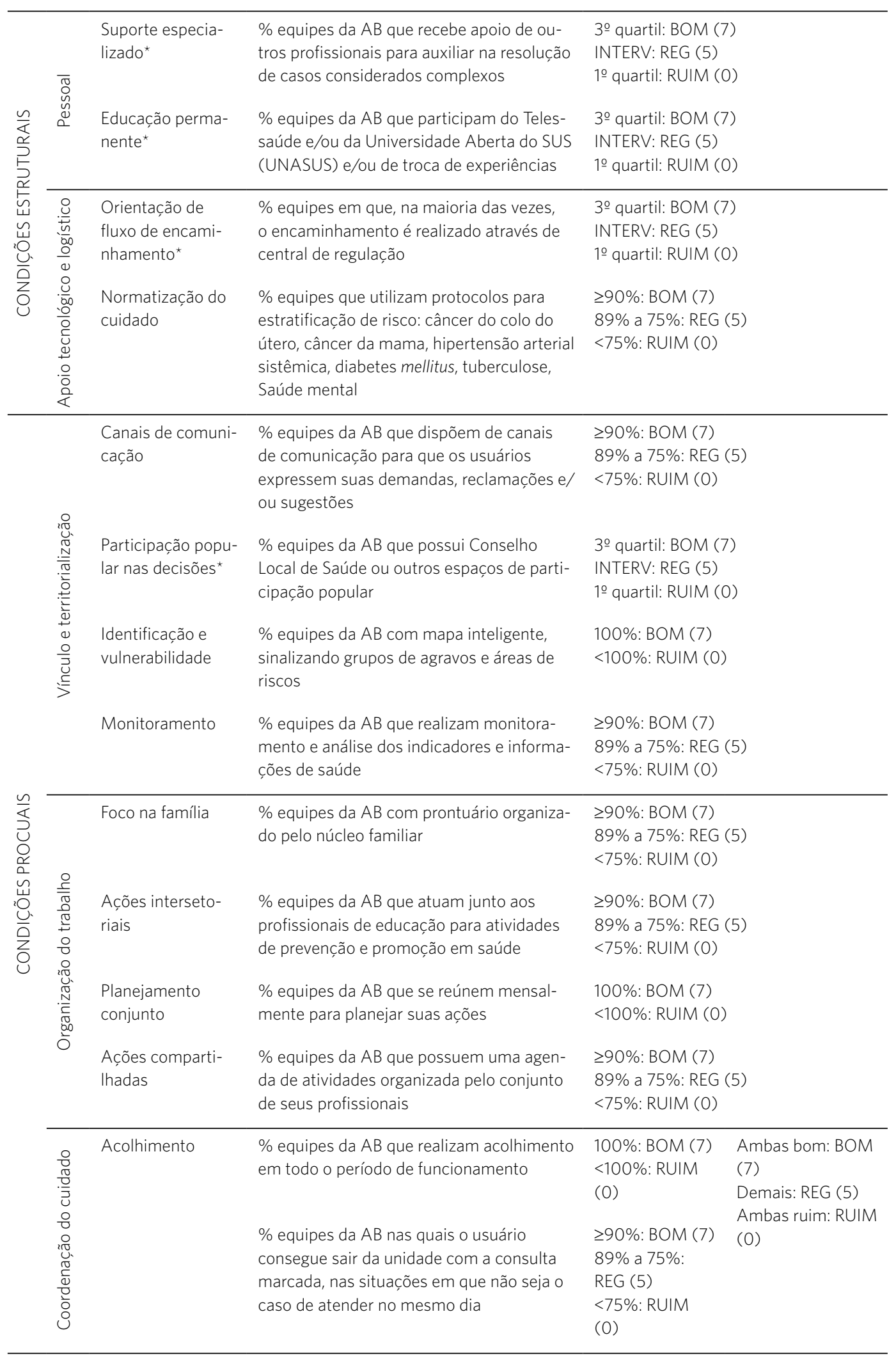


Quadro 1. (cont.)

\begin{tabular}{|c|c|c|c|c|}
\hline \multirow{3}{*}{ 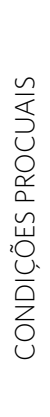 } & \multirow{3}{*}{$\begin{array}{l}0 \\
\frac{0}{0} \\
\frac{\pi}{0} \\
. \bar{J} \\
0 \\
0 \\
0 \\
0 \\
\frac{0}{0} \\
\frac{0}{0} \\
\frac{0}{0} \\
\frac{0}{0} \\
0 \\
0\end{array}$} & Encaminhamento & $\begin{array}{l}\text { \% equipes da } A B \text { responsáveis pelo agen- } \\
\text { damento da consulta do usuário com } \\
\text { especialista }\end{array}$ & 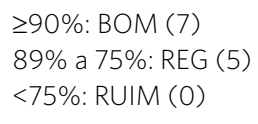 \\
\hline & & Acompanhamento & $\begin{array}{l}\text { \% equipes da } A B \text { que obtêm retorno da } \\
\text { avaliação realizada pelos especialistas dos } \\
\text { usuários encaminhados }\end{array}$ & $\begin{array}{l}\geq 90 \%: \text { BOM ( } 7) \\
89 \% \text { a } 75 \%: \text { REG (5) } \\
<75 \%: \text { RUIM (0) }\end{array}$ \\
\hline & & Responsabilização & $\begin{array}{l}\text { \% equipes da AB que realizam busca ativa } \\
\text { de exame citopatológico com atraso; pré- } \\
\text {-natal; com calendário vacinal atrasado; } \\
\text { hipertensos e diabéticos }\end{array}$ & $\begin{array}{l}\geq 90 \%: \text { BOM (7) } \\
89 \% \text { a } 75 \%: \text { REG (5) } \\
<75 \%: \text { RUIM (0) }\end{array}$ \\
\hline
\end{tabular}

REG = REGULAR; INTERV = INTERVALO.

* Parâmetros definidos segundo a distribuição quartil para cada estrato de porte populacional.

** Parâmetro definido conforme Portaria no 1007/2010 (BRASIL, 2010), para classificação de incentivos financeiros.

O modelo avaliativo construído e validado na primeira etapa orientou a classificação dos municípios catarinenses quanto à gestão municipal para o processo de trabalho das equipes da AB. Foram utilizados os dados secundários do segundo ciclo da avaliação externa do PMAQ-AB, coletados no ano de 2013, e cujo acesso encontra-se à disposição das universidades para análise. Foram consultadas outras bases de dados de informação secundárias disponíveis no site do Departamento de Informática do SUS (Datasus), de domínio público, conforme necessidade apontada na matriz avaliativa.

As informações obtidas sobre as equipes foram agregadas no nível municipal para emissão de juízo de valor. Os municípios foram analisados entre seus pares, segundo porte populacional, e a emissão de juízo de valor foi orientada pelos parâmetros definidos na matriz de análise e julgamento acordada nas oficinas de consenso (quadros 1 e 2).

Quadro 2. Matriz de julgamento da gestão municipal do processo de trabalho na Atenção Básica em saúde

Matriz avaliativa

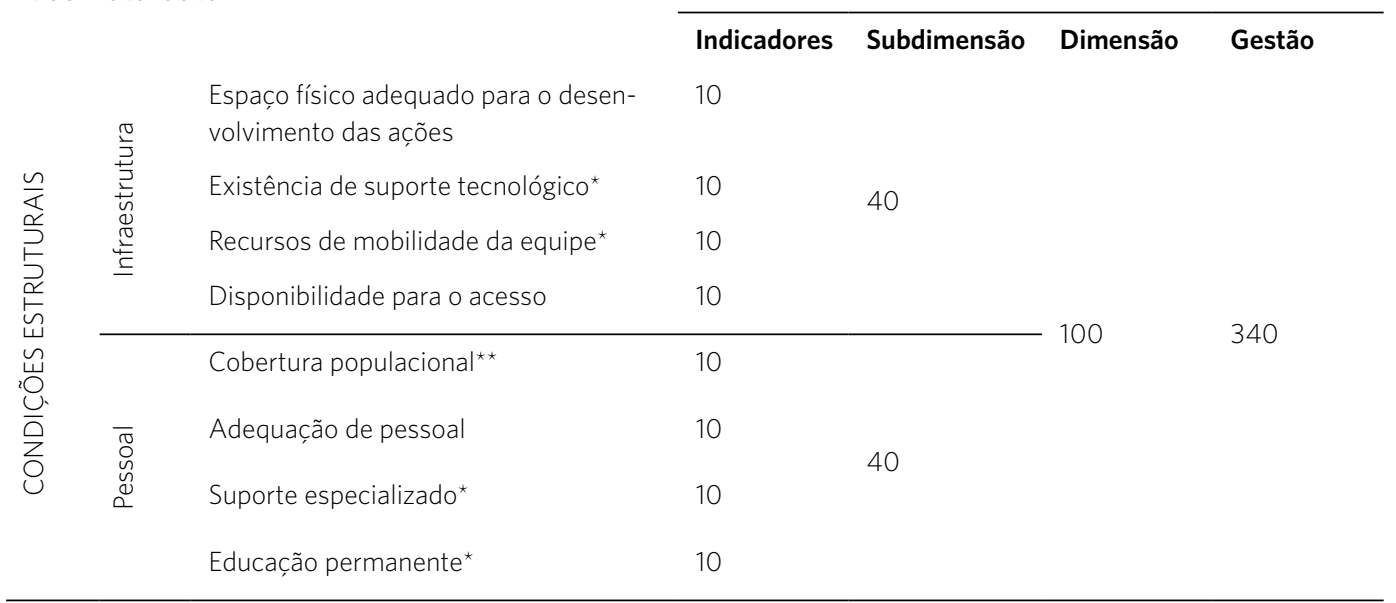


Quadro 2. (cont.)

\begin{tabular}{|c|c|c|c|c|c|}
\hline & 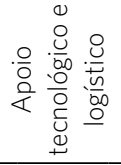 & $\begin{array}{l}\text { Orientação de fluxo de encaminha- } \\
\text { mento* } \\
\text { Normatização do cuidado }\end{array}$ & $\begin{array}{l}10 \\
10\end{array}$ & 20 & \\
\hline \multirow{3}{*}{ 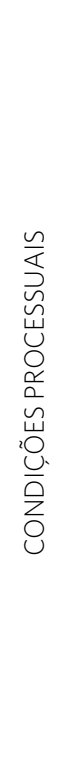 } & 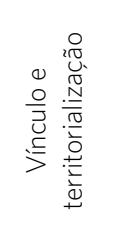 & $\begin{array}{l}\text { Canais de comunicação } \\
\text { Participação popular nas decisões* } \\
\text { Identificação e vulnerabilidade } \\
\text { Monitoramento }\end{array}$ & $\begin{array}{l}20 \\
20 \\
20 \\
20\end{array}$ & 80 & \multirow{3}{*}{240} \\
\hline & 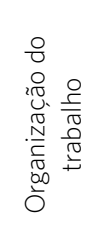 & $\begin{array}{l}\text { Foco na família } \\
\text { Ações intersetoriais } \\
\text { Planejamento conjunto } \\
\text { Ações compartilhadas }\end{array}$ & $\begin{array}{l}20 \\
20 \\
20 \\
20\end{array}$ & 80 & \\
\hline & 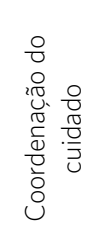 & $\begin{array}{l}\text { Acolhimento } \\
\text { Encaminhamento } \\
\text { Acompanhamento } \\
\text { Responsabilização }\end{array}$ & $\begin{array}{l}20 \\
20 \\
20 \\
20\end{array}$ & 80 & \\
\hline
\end{tabular}

* Parâmetros definidos segundo a distribuição quartil para cada estrato de porte populacional.

** Parâmetro definido conforme Portaria no 1007/2010 (BRASIL, 2010), para classificação de incentivos financeiros.

A MAJ possibilitou a análise da gestão municipal nos diferentes elementos avaliativos, de modo a identificar pontos de fragilidade e potencialidades. Para o julgamento das subdimensões, dimensões e da gestão, adotou-se o somatório dos pontos observados, confrontados com o potencial esperado (somatório do valor observado multiplicado por cem e dividido pelo somatório do valor esperado). O município foi classificado como 'bom' quando atingiu, pelo menos, $80 \%$ do valor esperado; como 'ruim' quando alcançou, no máximo, 50\%; e como 'regular' nas demais situações. Em função da dimensão 'Condições Processuais' ser fundamental para o objeto avaliado, os valores atribuídos aos seus elementos de análise foram superiores aos da dimensão 'Condições Estruturais'.

$\mathrm{Na}$ apresentação do resultado dos municípios, foi utilizado o software TabWin
$32.0^{\circledR}$, disponibilizado pelo Ministério da Saúde para a construção dos mapas de distribuição espacial, com a classificação dos municípios catarinenses, segundo as dimensões 'Condições Estruturais' e 'Condições Processuais', além do julgamento de valor da gestão municipal para o processo de trabalho na $\mathrm{AB}$.

Os dados foram processados em planilha no Microsoft Office Excel $^{\circledR}$, realizando-se análises conforme distribuição de frequências absolutas e relativas. Para os testes de associação, foi realizada análise bivariada por meio do teste exato de Fisher, considerando como nível de significância estatística o valor de $5 \%(\mathrm{p}<0,05)$.

A base de informações deste estudo é composta por dados secundários que já se encontram em domínio público, dispensando a aprovação pelo Comitê de Ética. 


\section{Resultados}

Os municípios participantes desta pesquisa representam $91,9 \%$ do total de municípios do estado de Santa Catarina. De acordo com o Instituto Brasileiro de Geografia e Estatística (IBGE), são, em sua maioria, de pequeno porte (IBGE, 2014).

A classificação dos municípios está exposta na tabela 1. A gestão municipal do processo de trabalho na $A B$ foi classificada como 'boa' em 10,7\% dos municípios catarinenses. Entre as duas dimensões analisadas, destacam-se as 'Condições Estruturais', que tratam de elementos de suporte e de apoio fornecidos pela gestão para que o processo de trabalho ocorra de acordo com o preconizado e esperado na AB. Nesta dimensão, 23,3\% dos municípios foram avaliados como 'bom', quase o dobro da outra dimensão, que analisa 'Condições Processuais'.

Tabela 1. Número e percentual de municípios, segundo a classificação na avaliação da gestão municipal do processo de trabalho na Atenção Básica em saúde, para indicadores, subdimensões, dimensões e gestão

\begin{tabular}{|c|c|c|c|c|c|c|}
\hline \multirow{3}{*}{$\begin{array}{l}\text { Dimensão } \\
\text { SUBDIMENSÃO } \\
\text { Indicadores }\end{array}$} & \multicolumn{6}{|c|}{ Classificação } \\
\hline & \multicolumn{2}{|c|}{ RUIM } & \multicolumn{2}{|c|}{ REGULAR } & \multicolumn{2}{|c|}{ Вом } \\
\hline & $\mathbf{N}$ & $\%$ & $\mathbf{N}$ & $\%$ & $\mathbf{N}$ & $\%$ \\
\hline Condições estruturais & 70 & 25,8 & 138 & 50,9 & 63 & 23,3 \\
\hline INFRAESTRUTURA & 85 & 31,4 & 100 & 36,9 & 86 & 31,7 \\
\hline $\begin{array}{l}\text { Espaço físico adequado para desenvolvi- } \\
\text { mento das ações }\end{array}$ & 23 & 8,5 & 150 & 55,3 & 98 & 36,9 \\
\hline Existência de suporte tecnológico & 60 & 22,1 & 16 & 5,9 & 195 & 72 \\
\hline Recursos de mobilidade da equipe & 46 & 17 & 17 & 6,3 & 208 & 76,7 \\
\hline Disponibilidade para o acesso & 39 & 14,4 & 198 & 73,1 & 34 & 12,5 \\
\hline PESSOAL & 79 & 29,2 & 115 & 42,4 & 77 & 28,4 \\
\hline Cobertura populacional & 106 & 39,1 & - & - & 165 & 60,9 \\
\hline Adequação de pessoal & 116 & 42,8 & 4 & 1,5 & 151 & 55,7 \\
\hline Suporte especializado & 40 & 14,8 & - & - & 231 & 85,2 \\
\hline Educação permanente & 43 & 15,9 & 6 & 2,2 & 222 & 81,9 \\
\hline APOIO TECNOLÓGICO E LOGÍSTICO & 223 & 82,3 & 8 & 2,9 & 40 & 14,8 \\
\hline Orientação de fluxo de encaminhamento & 66 & 24,3 & 33 & 12,2 & 172 & 63,5 \\
\hline Normatização do cuidado & 204 & 75,3 & 11 & 4 & 56 & 20,7 \\
\hline Condições processuais & 101 & 37,3 & 135 & 49,8 & 35 & 12,9 \\
\hline VÍNCULO E TERRITORIALIZAÇÃO & 41 & 15,1 & 95 & 35,1 & 135 & 49,8 \\
\hline Canais de comunicação & 30 & 11,1 & 10 & 3,7 & 231 & 85,2 \\
\hline Participação popular nas decisões & 59 & 21,8 & 77 & 28,4 & 135 & 49,8 \\
\hline Identificação de vulnerabilidade & 34 & 12,5 & - & - & 237 & 87,5 \\
\hline
\end{tabular}




\begin{tabular}{|c|c|c|c|c|c|c|}
\hline Monitoramento & 48 & 17,7 & 27 & 10 & 196 & 72,3 \\
\hline ORGANIZAÇÃO DO TRABALHO & 195 & 72 & 57 & 21 & 19 & 7 \\
\hline Foco na família & 43 & 15,9 & 9 & 3,3 & 219 & 80,8 \\
\hline Ações intersetoriais & 236 & 87,1 & 3 & 1,1 & 32 & 11,8 \\
\hline Planejamento conjunto & 82 & 30,3 & - & - & 189 & 69,7 \\
\hline Ações compartilhadas & 189 & 69,7 & 20 & 7,4 & 62 & 22,9 \\
\hline COORDENAÇÃO DO CUIDADO & 196 & 72,3 & 56 & 20,7 & 19 & 7 \\
\hline Acolhimento & 5 & 1,9 & 77 & 28,4 & 189 & 69,7 \\
\hline Encaminhamento & 79 & 29,1 & 26 & 9,6 & 166 & 61,3 \\
\hline Acompanhamento & 214 & 79 & 10 & 3,7 & 47 & 17,3 \\
\hline Responsabilização & 204 & 75,3 & 19 & 7 & 48 & 17,7 \\
\hline $\begin{array}{l}\text { Gestão do processo de trabalho na Aten- } \\
\text { ção Básica em saúde }\end{array}$ & 86 & 31,7 & 156 & 57,6 & 29 & 10,7 \\
\hline
\end{tabular}

Uma análise detalhada das 'Condições Estruturais' permite observar melhor resultado na subdimensão 'Infraestrutura' (31,7\%), com destaque para 'Recursos de Mobilidade da Equipe' e 'Existência de Suporte Tecnológico', que apresentaram mais de 70\% dos municípios avaliados como 'bom'. Na 'Disponibilidade para o Acesso', que considerou o horário de funcionamento das Unidades Básicas de Saúde (UBSs) por, no mínimo, 40 horas semanais e a disponibilidade de, pelo menos, uma UBS no município atuar com horário estendido, não foi observado comportamento tão satisfatório.

'Suporte Especializado' e 'Educação Permanente' foram destaques na análise de 'Pessoal', com mais de $80 \%$ dos municípios avaliados como 'bom'. Em contrapartida, no indicador 'Adequação de Pessoal', uma parcela significativa de municípios $(42,8 \%)$ ainda não possui profissionais da saúde bucal (dentista e auxiliar de saúde bucal) em todas as EqAB.
O pior desempenho na análise estrutural foi no 'Apoio Tecnológico e Logístico', com menos de $14,8 \%$ dos municípios classificados como 'bom'. Nesta subdimensão, destaca-se o resultado alcançado no indicador 'Normatização do Cuidado', que se refere à utilização de protocolos para a estratificação de riscos em agravos elencados como prioritários na $\mathrm{AB}$, com $75,3 \%$ dos municípios classificados como 'ruim'.

A segunda dimensão do estudo analisa aspectos da organização do processo de trabalho, com foco no indivíduo/família, reconhecendo seu ambiente físico e social de forma integral e contínua, e em conformidade com os princípios do SUS, expressando 'Condições Processuais' com apenas 12,9\% dos municípios classificados como 'bom'. Nesta, chamam a atenção a 'Organização do Trabalho' e a 'Coordenação do Cuidado', com aproximadamente $90 \%$ dos munícipios classificados como 'ruim' e 'regular'.

Na 'Organização do Trabalho', as ‘Ações 
Intersetoriais' e as 'Ações Compartilhadas' não ocorrem como o preconizado em $87,1 \%$ e $69,7 \%$ dos elementos analisados, respectivamente. A situação agrava-se na análise da 'Coordenação do Cuidado', com destaque para o 'Acompanhamento' e a 'Responsabilização'. Apenas $17 \%$ dos municípios cumprem os requisitos de contrarreferência com outros níveis de atenção e realização de busca ativa para as situações hipertensos e diabéticos, pré-natal, exames citopatológico e vacinas em atraso.

$\mathrm{Na}$ tabela 2, observa-se que municípios menores apresentaram melhor classificação, tanto na análise das dimensões quanto na avaliação geral da gestão municipal. Aproximadamente metade dos municípios de até 5 mil habitantes destacaram-se com resultado 'bom' na dimensão 'Condições Estruturais', observando-se um declínio na classificação à medida que aumenta o porte populacional. Nas 'Condições Processuais', pouco mais de $25 \%$ dos municípios de menor porte populacional foram classificados como 'bom', enquanto $7 \%$ dos municípios com população acima de 25 mil obtiveram classificação semelhante (tabela 2).

Tabela 2. Avaliação da gestão municipal do processo de trabalho na Atenção Básica em saúde, segundo o porte populacional

\begin{tabular}{|c|c|c|c|c|c|c|c|c|}
\hline \multirow{2}{*}{$\begin{array}{l}\text { Classificação } \\
\text { № de habitantes }\end{array}$} & \multicolumn{2}{|c|}{ RUIM } & \multicolumn{2}{|c|}{ REGULAR } & \multicolumn{2}{|c|}{ BOM } & \multicolumn{2}{|c|}{ TOTAL } \\
\hline & $\mathbf{N}$ & $\%$ & $\mathbf{N}$ & $\%$ & $\mathbf{N}$ & $\%$ & $\mathbf{N}$ & $\%$ \\
\hline Gestão municipal & \multicolumn{8}{|c|}{$<0,001^{\star}$} \\
\hline Até 5 mil habitantes & 6 & 6,8 & 60 & 68,2 & 22 & 25 & 88 & 100 \\
\hline De 5 a 10 mil habitantes & 16 & 31,4 & 33 & 64,7 & 2 & 3,9 & 51 & 100 \\
\hline De 10 a 25 mil habitantes & 29 & 38,7 & 45 & 60 & 1 & 1,36 & 75 & 100 \\
\hline Acima de 25 mil habitantes & 35 & 61,4 & 18 & 31,6 & 4 & 7 & 57 & 100 \\
\hline Condições estruturais & \multicolumn{8}{|c|}{$<0,001^{\star}$} \\
\hline Até 5 mil habitantes & 5 & 5,7 & 40 & 45,4 & 43 & 48,9 & 88 & 100 \\
\hline De 5 a 10 mil habitantes & 11 & 21,6 & 28 & 54,9 & 12 & 23,5 & 51 & 100 \\
\hline De 10 a 25 mil habitantes & 18 & 24 & 51 & 68 & 6 & 8 & 75 & 100 \\
\hline Acima de 25 mil habitantes & 36 & 63,2 & 19 & 33,3 & 2 & 3,5 & 57 & 100 \\
\hline Condições processuais & \multicolumn{8}{|c|}{$<0,001^{\star}$} \\
\hline Até 5 mil habitantes & 13 & 14,8 & 50 & 56,8 & 25 & 28,4 & 88 & 100 \\
\hline De 5 a 10 mil habitantes & 21 & 41,2 & 26 & 51 & 4 & 7,8 & 51 & 100 \\
\hline De 10 a 25 mil habitantes & 33 & 44 & 40 & 53,3 & 2 & 2,7 & 75 & 100 \\
\hline Acima de 25 mil habitantes & 34 & 59,7 & 19 & 33,3 & 4 & 7 & 57 & 100 \\
\hline
\end{tabular}

*Valor-p utilizando teste exato de Fisher. 
Na distribuição espacial, observa-se que municípios do oeste catarinense obtiveram melhores classificações do que os municípios das regiões Central e Litorânea. Menos de $20 \%$ dos municípios do Oeste foram avaliados como 'ruins' na dimensão 'Condições Estruturais', enquanto nas regiões Central e Litorânea esse percentual aumentou para $35,7 \%$ e $45,7 \%$, respectivamente. Nas 'Condições Processuais', a região Litorânea manteve comportamento idêntico ao da dimensão estrutural, diferente das regiões Oeste e Central, que sofreram pequenas oscilações (figura 1).

Figura 1. Avaliação da gestão municipal do processo de trabalho na Atenção Básica em saúde

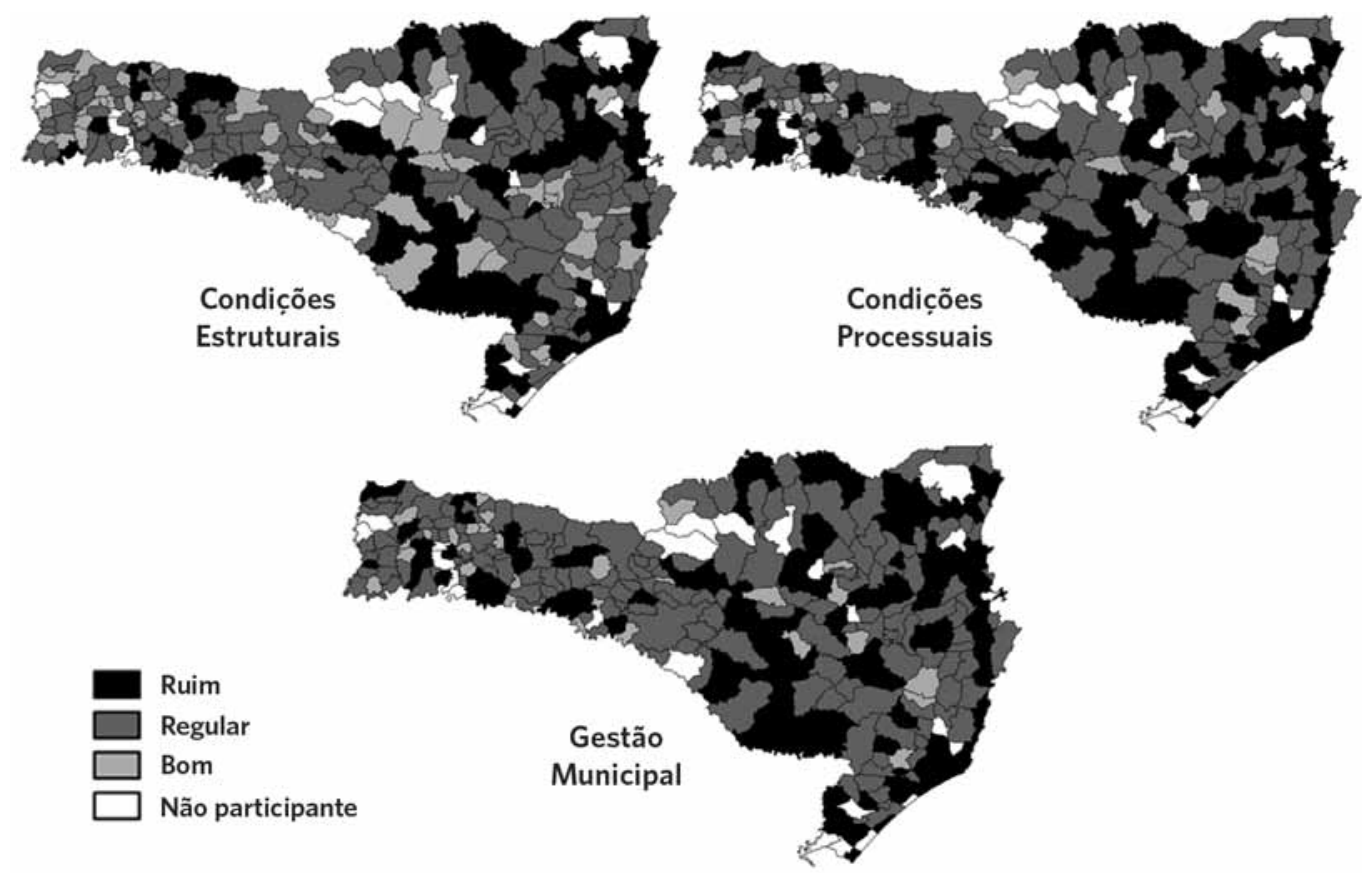

\section{Discussão}

A aplicação em Santa Catarina justifica-se por ser este um dos estados de maior adesão dos municípios e equipes ao PMAQ, nos dois ciclos de análise do Programa. No ciclo 2, base de dados deste estudo, a adesão municipal foi de 99,3\%, incluindo 91,6\% das equipes existentes no estado. Isso permitiu a agregação dos resultados das equipes por município e uma análise regional. Sua aplicação mostrou-se viável e independente do porte populacional. Os melhores resultados, em municípios de menor porte populacional, também foram encontrados nos estudos de Colussi e Calvo (2011) e Lacerda et al. (2012), além da maior proporção destes municípios na região do oeste catarinense.

Melhores resultados das 'Condições Estruturais' podem indicar um comprometimento da gestão municipal com a superação de problemas estruturais evidenciados ao longo da consolidação da AB. Resultados semelhantes foram encontrados em estudo que analisou condições estruturais da $\mathrm{AB}$ em municípios de pequeno porte, majoritários no universo aqui analisado (PIMENTEL; ALBUQUERQUE; SOUZA, 2015). 
'Recursos de Mobilidade das Equipes' e 'Existência de Suporte Tecnológico' foram os aspectos assegurados pelo maior número de gestores municipais, aferidos pela disponibilidade de veículo que atenda às necessidades das equipes e de computador com acesso à Internet em, pelo menos, um consultório. $\mathrm{Na}$ literatura, foram encontrados achados contraditórios, em especial, nas regiões Sudeste e Nordeste. Identificaram-se problemas como a falta de computadores conectados à Internet para a transmissão de informações, e carência na oferta de veículos para a realização de visitas domiciliares e atividades de educação em saúde nas comunidades (PROTÁSIO ET AL., 2014; GARCIA ET AL., 2014). Estudo mais recente identificou acentuadas desigualdades regionais, com as regiões Norte e Nordeste apresentando os piores resultados e as regiões Centro-Oeste, Sul e Sudeste com os maiores percentuais de adequação da estrutura das unidades de saúde (TOMASI ET AL., 2015).

O ponto crítico nesse bloco de avaliação foi 'Disponibilidade para o Acesso', ao demonstrar que a $\mathrm{AB}$, como entrada preferencial do sistema, possui barreiras decorrentes dos horários de funcionamento das unidades. Observou-se que os municípios não asseguram o cumprimento das 40 horas semanais nem possuem, pelo menos, uma unidade no município com horário estendido para atender às necessidades da população, com vistas a diminuir barreiras de acesso e melhorar a utilização dos serviços (BRASIL, 2011B). A dificuldade de acesso identificada impacta de forma negativa sobre os fundamentos e diretrizes propostos para a $\mathrm{AB}$, e contribui para o excesso de demanda dos prontos-socorros, que deveriam dedicar-se ao atendimento das urgências/emergências, o que aponta o horário de funcionamento adequado da unidade como item mais crítico (REIS ET AL., 2013; MOREIRA ET AL., 2015). Em Minas Gerais, a indisponibilidade da equipe para o atendimento ao usuário em horários noturnos e finais de semana resultou em baixa resolubilidade, principalmente, quando relacionada a eventos agudos (CHOMATAS ET AL., 2013).

A avaliação satisfatória do 'Suporte Especializado', seguido pela 'Educação Permanente' na análise da subdimensão 'Pessoal', demonstra a preocupação da gestão em estruturar suas equipes com profissionais especializados e qualificados, nos municípios estudados. Também no cenário nacional, as ações de educação permanente vêm se destacando no desenvolvimento do processo de trabalho das equipes da $A B$ (CARNUT; SILVA, 2011). A oferta de ações de educação permanente, com destaque para a participação no Telessaúde e nos cursos presenciais, foi também encontrada em estudo que analisou a gestão do trabalho na $\mathrm{AB}$, a partir das entrevistas realizadas pelo PMAQ no primeiro ciclo, mantendo aparentemente, a tendência no segundo ciclo (SEIDL ET AL., 2014).

Destaca-se a insuficiência de profissionais de saúde bucal nas equipes, embora a atenção nesta área seja indissociável dos demais serviços de saúde ofertados pela $\mathrm{AB}$. Uma análise comparativa entre as coberturas populacionais da Equipe de Saúde Bucal (EqSB) e da EqAB, em municípios pernambucanos, permitiu observar baixa cobertura populacional da EqSB, variando entre 30\% e 19,5\% (GODOI; MELLO; CAETANO, 2014). Nessa mesma perspectiva, Collussi e Calvo (2011), ao estudarem municípios catarinenses, constataram relação desfavorável entre o número de profissionais auxiliares e cirurgiões-dentistas.

$\mathrm{O}$ resultado do indicador 'Normatização do Cuidado' apresentou baixo desempenho entre os municípios estudados, sendo que o uso de protocolos clínicos para o atendimento previsto na Política Nacional de Atenção Básica (PNAB) propõe uma alternativa para a complexidade das tecnologias de cuidado produtoras de saúde (BRASIL, 2011B). Dados semelhantes foram encontrados em estudo baseado nos dados do primeiro ciclo do PMAQ-AB, com 75\% das unidades respondendo negativamente para a existência de protocolos (PROTÁSIO ET AL., 2014). 
Na dimensão 'Condições Processuais', o resultado alcançado pelos municípios foi insatisfatório, o que sugere um distanciamento, por parte da gestão e das equipes, das recomendações do processo de trabalho organizado com foco no indivíduo/família. Na subdimensão 'Vínculo e Territorialização', o resultado indica precariedade nas relações gestão-profissionais-usuários, principalmente, na 'Participação Popular nas Decisões'. Resultados semelhantes foram encontrados por Cruz et al. (2012), com indicação de que os profissionais desvalorizavam o espaço de diálogo como oportunidade de aprender com o saber popular, e a população utilizava o espaço de participação popular apenas como 'balcão de reclamações' sobre os atendimentos de saúde.

'Organização do Trabalho' e 'Coordenação do Cuidado' foram as subdimensões com os piores resultados, agravando a avaliação da gestão do processo de trabalho na $\mathrm{AB}$, em municípios catarinenses. $\mathrm{Na}$ 'Organização do Trabalho', destaque para indicadores de 'Ações Intersetoriais' e ‘Ações Compartilhadas'. A intersetorialidade é um dos princípios organizativos da $\mathrm{AB}$, e compreende a articulação e o envolvimento da saúde com outros setores, seja no planejamento de ações ou no alcance de metas comuns. As ações compartilhadas buscam respeitar o princípio da integralidade, além de possibilitar que todos os profissionais se mantenham responsáveis pelo cuidado e de qualificar o processo organizativo. Essa prática deve ser orientada pela gestão municipal, pois possibilita a troca de saberes entre os profissionais, evitando a fragmentação da assistência. Além das condições estruturais, é preciso garantir articulação entre os setores externos ao setor saúde, principalmente, os setores educação, planejamento urbano e segurança (SANTOS; RIGOTTO, 2010; PEREIRA, 2010).

A 'Coordenação do Cuidado' compreende a sincronicidade de ações de 'Acolhimento', 'Encaminhamento', 'Monitoramento' e 'Responsabilização', independentemente do ponto de atenção no qual o indivíduo esteja no sistema. A gestão deve dar condições para a equipe da AB ser capaz de coordenar as ações de saúde e prestar atenção à pessoa/família, mesmo em outros níveis de atenção, atuando de forma integrada com os profissionais dos serviços especializados que compõem a rede de saúde. No presente estudo, menos de $20 \%$ dos municípios foram classificados como 'bom' na análise do 'Acompanhamento' e da 'Responsabilização' do cuidado. Achados nacionais corroboram esse resultado mostrando que ações da coordenação do cuidado ainda são incipientes, com menos de $1 / 3$ das equipes declarando contatar o especialista para trocar informações e discutir casos relativos aos pacientes encaminhados (SEIDL ET AL., 2014).

Entre os desafios do SUS, estão a qualificação da $\mathrm{AB}$ para exercer a coordenação do cuidado e a reorganização dos pontos de atenção, intercomunicantes, capazes de assegurar que a linha de cuidado integral seja plenamente articulada e forneça aos usuários do SUS respostas adequadas às suas necessidades (BARATIERI; MARCON, 2011; OLIVEIRA; PEREIRA, 2013). Identifica-se que os aspectos estruturais, isoladamente, não garantem o desenvolvimento de um processo de trabalho com qualidade na $\mathrm{AB}$. Os profissionais e gestores municipais de saúde precisam retomar o entendimento do processo de trabalho como um objeto contínuo do trabalho em equipe. Atualmente, ele é executado por meio de instrumentos fragmentados, que diminuem a qualidade dos serviços e comprometem o potencial de resolubilidade da $A B$ (CORDEIRO; SOARES, 2015).

\section{Conclusão}

O estudo identificou alguns avanços da gestão municipal do processo de trabalho na $\mathrm{AB}$, principalmente, nas condições estruturais proporcionadas às EqAB. Foi possível identificar que a maior parte das $\mathrm{EqAB}$ 
estudadas possuem acesso ao aporte tecnológico, condição de mobilidade para a equipe, suporte especializado e educação permanente. Porém, importantes desafios foram identificados nas condições processuais.

Segundo os dados encontrados, instrumentos de participação popular na gestão local, desenvolvimento de ações intersetoriais e compartilhadas, acompanhamento e responsabilização do cuidado, em todos os pontos de atenção, na perspectiva de um trabalho articulado, ainda são elementos a serem efetivados no estado de Santa Catarina, e a coincidência com achados da literatura nos permitem afirmar que, também, no País. Isto remete à reflexão de que os problemas não se concentram apenas na disponibilidade de recursos, mas também na formação profissional, que, apesar dos recentes esforços de mudança, com ajustes curriculares, ainda não atendem às necessidades do SUS.

\section{Referências}

BARATIERI, T.; MARCON, S. S. Longitudinalidade do cuidado: compreensão dos enfermeiros que atuam na estratégia saúde da família. Esc. Anna Nery Rev. Enferm., Rio de Janeiro, v. 15, n .4, p. 802-810, 2011.

BRASIL. Ministério da Saúde. Portaria nº 1.007, de 04 de maio de 2010. Define critérios para regulamentar a incorporação do Agente de Combate às Endemias

- ACE, ou dos agentes que desempenham essas atividades, mas com outras denominações, na atenção primária à saúde para fortalecer as ações de vigilância
O PMAQ-AB, inserido no escopo de políticas indutoras do Ministério da Saúde e aliado a outras fontes de financiamento, pode ter produzido melhorias estruturais na $\mathrm{AB}$ e contribuído com os resultados encontrados. Ações que possam tornar a $A B$ ordenadora do cuidado em saúde, proporcionando acesso equânime e processo de trabalho consoante com os pressupostos da atenção primária em saúde, são prementes em municípios com distintas realidades populacionais e de organização dos serviços.

\section{Colaboradores}

Karine Cardoso Fontana e Patrícia Maria de Oliveira Machado desenvolveram a pesquisa, a análise de dados e a elaboração do manuscrito. Josimari Telino de Lacerda orientou a pesquisa, a concepção do manuscrito e fez a revisão crítica do mesmo. em saúde junto às equipes de Saúde da Família. Diário Oficial [da] União. Brasília, DF: Ministério da Saúde, 2010. Disponível em: <http://bvsms.saude.gov.br/ bvs/saudelegis/gm/2010/prt1007_04_05_2010.html>. Acesso em: 29 ago. 2016.

Ministério da Saúde. Portaria n ${ }^{\circ} 1.654$, de 19 de julho de 2011. Institui, no âmbito do Sistema Único de Saúde, o Programa Nacional de Melhoria do Acesso e da Qualidade da Atenção Básica (PMAQ-AB) e o Incentivo Financeiro do PMAQ-AB, denominado 
Componente de Qualidade do Piso de Atenção Básica Variável - PAB Variável. Diário Oficial [da] União. Brasília, DF: Ministério da Saúde, 201la. Disponível em: <http://bvsms.saude.gov.br/bvs/saudelegis/gm/2011/ prt1654_19_07_2011.html>. Acesso em: 29 ago. 2016.

Ministério da Saúde. Portaria n ${ }^{\circ} 2.488$, de 21 de outubro de 2011. Aprova a Política Nacional de Atenção Básica, estabelecendo a revisão de diretrizes e normas para a organização da Atenção Básica, para a Estratégia Saúde da Família (ESF) e o Programa de Agentes Comunitários de Saúde (PACS). Diário Oficial [da] União, Brasília, DF: Ministério da Saúde, 2011b. Disponível em: <http://bvsms.saude.gov.br/bvs/ saudelegis/gm/2011/prt2488_21_10_2011.html>. Acesso em: 29 ago. 2016.

CARNUT, L.; SILVA, J. L. Saúde bucal na estratégia de saúde da família: das desigualdades sociais à inserção das ações. J. Manag. Prim. Health Care, Londres, v. 3, n. 2, p. 186-196, 2011.

CHOMATAS, E. et al. Avaliação da presença e extensão dos atributos da atenção primária em Curitiba. Rev. Bras. Med. Fam. Comunidade, Rio de Janeiro, v. 8, n. 29, p. 294-303, 2013.

COLUSSI, C.; CALVO, M. C. M. Modelo de avaliação da saúde bucal na atenção básica. Cad. Saúde Pública, Rio de Janeiro, v. 1, n. 27, p. 1731-1745, 2011.

CORDEIRO, L.; SOARES, C. B. Processo de trabalho na Atenção Primária em Saúde: pesquisa-ação com Agentes Comunitários de Saúde. Ciênc. Saúde Col., Rio de Janeiro, v. 20, n. 11, p. 3581-3588, 2015.

CRUZ, P. J. S. C. et al. Desafios para a participação popular em saúde: reflexões a partir da educação popular na construção de conselho local de saúde em comunidades de João Pessoa, PB. Saúde Soc., São Paulo, v. 21, n. 4 , p. 1087-1100, 2012.

FERREIRA, V. L. As múltiplas faces da gestão: a prática gerencial nos serviços de saúde de Piraí. 2012. 101 f. Dissertação (Mestrado em Medicina Social). Rio de Janeiro, Universidade do Estado do Rio de Janeiro, 2012.
GARCIA, A. C. P. et al. Análise da organização da atenção básica no Espírito Santo: (des)velando cenários. Saúde em Debate, Rio de Janeiro, v. 38, n. esp., p. 221-236, out. 2014.

GODOI, H.; MELLO, A. L. S. F.; CAETANO, J. C. Rede de atenção à saúde bucal: organização em municípios de grande porte de Santa Catarina, Brasil. Cad. Saúde Pública, Rio de Janeiro, v. 30, n. 2, p. 318-332, 2014.

\section{INSTITUTO BRASILEIRO DE GEOGRAFIA E} ESTATÍSTICA (IBGE).Estados@: Santa Catarina. Brasília, DF: IBGE, 2014. Disponível em: <http://www. ibge.gov.br/estadosat/perfil.php?sigla=sc $>$. Acesso em: 14 abr. 2015

LACERDA, J. T. et al. Avaliação da gestão para o planejamento em saúde em municípios catarinenses. Ciênc. Saúde Col., Rio de Janeiro, v. 17, n. 4, p. 851-859, 2012

MOREIRA, R. N. et al. Qualidade de serviços: análise de uma unidade do Programa Saúde da Família. Gestão Saúde, Brasília, DF, v. 6, n. 2, p. 1267-1288, 2015.

OLIVEIRA, M. A. C.; PEREIRA, I. C. Atributos essenciais da atenção primária e a estratégia saúde da família. Rev. Bras. Enferm., Brasília, DF, v. 66, n. esp., p. 158-164, 2013

PAIM, J. Modelos de atenção à saúde no Brasil. In: GIOVANELLA, L. et al. (Org.). Políticas e sistema de saúde no Brasil. Rio de Janeiro: Fiocruz, 2008. p. 547-574.

PAIM, J. et al. The Brazilian health system: history, advances, and challenges. The Lancet, Londres, v. 377, n. 9779, p. 1778-1797, 2011.

PEREIRA, R. R. Plano diretor como instrumento para promoção da saúde: um estudo de caso em Nova Friburgo (RJ). Rev. Baiana Saúde Pública, Salvador, v. 34, n. 3, p. 612-622, 2010.

PIMENTEL, F. C.; ALBUQUERQUE, P. C.; SOUZA,

W. V. A Estratégia Saúde da Família no estado de Pernambuco: avaliação da estrutura das equipes por 
porte populacional. Saúde em Debate, Rio de Janeiro, v. 39, n. 104, p. 88-101, 2015.

PROTASIO, A. P. L. et al. Avaliação do sistema de referência e contrarreferência do estado da Paraíba segundo os profissionais da Atenção Básica no contexto do $1^{\circ}$ ciclo de avaliação externa do PMAQ-AB. Saúde em Debate, Rio de Janeiro, v. 38, n. esp., p. 209-220, out. 2014.

REIS, R. S. et al. Acesso e utilização dos serviços na estratégia saúde da família na perspectiva dos gestores, profissionais e usuários. Ciênc. Saúde Col., Rio de Janeiro, v. 18, n. 11, p. 3321-3331, 2013.

SANTOS, A. L.; RIGOTTO, R. M. Território e territorialização: incorporando as relações produção, trabalho, ambiente e saúde na atenção básica à saúde. Trab. Educ. Saúde, Rio de Janeiro, v. 8, n. 3, p. 387-406, 2010.
SEIDL, H. et al. Gestão do trabalho na atenção básica em saúde: uma análise a partir da perspectiva das equipes participantes do PMAQ-AB. Saúde em Debate, Rio de Janeiro, v. 38, n. esp., p. 94-108, out. 2014.

SUMAR, N.; FAUSTO, M. C. R. Atenção primária à saúde: a construção de um conceito ampliado. $J$. Manag. Prim. Health Care, Londres, v. 5, n. 2, p. 202212, 2014.

TOMASI, E. et al. Estrutura e processo de trabalho na prevenção do câncer de colo de útero na atenção básica à saúde no Brasil: Programa de Melhoria do Acesso e da Qualidade - PMAQ. Rev. Bras. Saúde Mater. Infantil, Recife, v. 15, n. 2, p. 171-180, 2015.

\footnotetext{
Recebido para publicação em abril de 2016

Versão final em agosto de 2016

Conflito de interesses: inexistente

Suporte financeiro: não houve
} 